\title{
Health Care Professional's Practice in Cancer Prevention: Smoking Cessation Counseling as an Example
}

\author{
Hsiu-Wen Tsai ${ }^{1}$, Yeur-Hur Lai ${ }^{2}$, Hsein-Lin Wu ${ }^{3}$, Kuang-Chieh Hsueh ${ }^{4}$
}

\author{
${ }^{1}$ Hsin-Sheng Junior College of Medical Care and Management, Taoyuan, Taiwan \\ ${ }^{2}$ School of Nursing, College of Medicine, National Taiwan University, Taipei, Taiwan \\ ${ }^{3}$ Department of Thoracic Medicine, Taiwan Adventist Hospital, Taipei, Taiwan \\ ${ }^{4}$ Departments of Family Medicine, Kaohsiung Veterans General Hospital, Kaohsiung, Taiwan
}

\section{Introduction :}

Smoking cessation is one of the most important issues in cancer prevention. In Taiwan, a 3-level (basic, intermediate, advanced) 48-hour smoking cessation educators (SCE) training programs has been conducted nationally by government. It is unclear what the current status of SCE trainees' practice of SC counseling before they received the comprehensive SCE training.

Objectives: (1) the implementation of SC counseling in SCE trainees, and (2) identify factors associated with SCE trainees' applying of counseling in SC.

\section{Methods:}

This study is part of a major project. A crosssectional survey was conducted among SCE trainee $(\mathrm{N}=250)$. The survey includes levels of SC

counseling and attitudes toward SC practice. Data 1 were analyzed by descriptive statistics and Pearson's correlation..

\section{Results:}

The mostly applied SC counseling activities were as follow: "Provide resources and assistance", "Ask tobacco users if they are willing to quit", "Identify reasons for quitting". However, requiring more knowledge and skills, such as "set a quit date", "refer to web resources", "use cessation materials that are appropriate by age, culture, language, education, and pregnancy status" are less implemented. The correlation results shows that positive correlation between SC counseling and perceived responsibility $(\mathrm{r}=.39, \mathrm{p}<.01)$ and selfefficacy in SC $(r=.23, p<.01)$.

Table 1. Correlation among scores for counseling, responsibility, and self-efficicy $(\mathrm{N}=250)$

\section{SCC Responsibility Self-efficicy}

$\mathrm{SCC}^{1} \quad 1.000$

Responsibility $^{2} \quad .392 * *$

1.000

Self-efficicy ${ }^{3}$

$.232 * *$

$.427 * *$

1.000

${ }^{1}$. SCC: smoking cessation counseling intervention

2.Responsibility: Belief of helping people to stop smoking as the part of their responsibilities.

${ }^{3}$ Self-efficacy: confidence in ability to help people stop smoking.

Note: $* * \mathrm{P}<.01$
Table 2. Means and standard deviations of the smoking cessation counseling intervention $(\mathrm{N}=250)$

\begin{tabular}{|l|l|}
\hline \multicolumn{1}{|c|}{ Items } & Mean(SD) \\
\hline $\begin{array}{l}\text { If tobacco users are willing to quit, I provide resources and } \\
\text { assistance }\end{array}$ & $\mathbf{2 . 8 8}(.85)$ \\
\hline I ask tobacco users if they are willing to quit & $\mathbf{2 . 8 1}(.85)$ \\
\hline I provide the number for the toll-free National Quitline & $\mathbf{2 . 7 7 ( . 9 4 )}$ \\
\hline I advise tobacco users to quit & $\mathbf{2 . 7 7 ( . 8 7 )}$ \\
\hline I identify reasons for quitting and benefits of quitting & $\mathbf{2 . 7 6 ( . 8 5 )}$ \\
\hline I assess my patient tobacco use & $\mathbf{2 . 7 5 ( . 9 3 )}$ \\
\hline I document my patient tobacco use & $\mathbf{2 . 5 3 ( 1 . 0 )}$ \\
\hline $\begin{array}{l}\text { I advise patients that having other smokers in household } \\
\text { hinders successful quitting }\end{array}$ & $\mathbf{2 . 5 2 ( . 9 4 )}$ \\
\hline
\end{tabular}

If tobacco users are not willing to quit, I provide resources and $\quad \mathbf{2 . 4 6}(.91)$ help patient identify barriers to quitting

I refer the patient to web resources for Agency for Healthcare Research and Quality

$2.43(.88)$

I advise smokers to get support from family, friends, and coworkers

I advise patients that drinking alcohol is strongly associated with relapse

I advise patients that total abstinence is essential- not even a single puff

I advise patients if relapse occurs, they should review the circumstances and learn from the experience

$2.42(.89)$

$2.32(.93)$

$2.29(.99)$

$2.24(.92)$

I help patients anticipate nicotine withdraw

$2.23(.87)$

I advise patients if relapse occurs, they should repeat the quit attempt it is part of the quitting process

2.22(.86)

I recommend use of over-the-counter nicotine patch, gum, or lozenge; or get a prescription for nasal spray, inhaler, or buproprion SR unless contraindicated

I advise patients that if relapse occurs, they should reassess the pharmacotherapy use and problems

I provide information for follow-up visits the patient's doctor

I help the patient anticipate challenges, particularly during the critical first few weeks

I review past quit attempts- what helped, what led to relapse

I use cessation materials that are appropriate by age, culture, language, education, and pregnancy status

I refer the patient to web resources for Tobacco Free Nurses Initiative

$2.17(.91)$

$2.15(.86)$

2.13(.86)

2.13(.81)

$2.08(.86)$

$1.95(.83)$

I advise smokers to set a quit date

$1.90(.85)$

\section{Conclusion :}

The results suggested that there will be important to apply SC education to increase the health care professional to apply SC counseling. Enhancing health care personnel's perceived responsibility and self-efficacy might also increase their willingness to apply SC counseling. 